\title{
On the self-adjointness of certain reduced Laplace-Beltrami operators
}

\author{
L. FEHÉR ${ }^{a}$ and B.G. PUSZTAI ${ }^{b}$ \\ ${ }^{a}$ Department of Theoretical Physics, MTA KFKI RMKI \\ 1525 Budapest 114, P.O.B. 49, Hungary, and \\ Department of Theoretical Physics, University of Szeged \\ Tisza Lajos krt 84-86, H-6720 Szeged, Hungary \\ e-mail: lfeher@rmki.kfki.hu \\ ${ }^{b}$ Centre de recherches mathématiques, Université de Montréal \\ C.P. 6128, succ. centre ville, Montréal, Québec, Canada H3C 3J7, and \\ Department of Mathematics and Statistics, Concordia University \\ 1455 de Maisonneuve Blvd. West, Montréal, Canada H3G 1M8 \\ e-mail: pusztai@CRM.UMontreal.CA
}

\begin{abstract}
The self-adjointness of the reduced Hamiltonian operators arising from the LaplaceBeltrami operator of a complete Riemannian manifold through quantum Hamiltonian reduction based on a compact isometry group is studied. A simple sufficient condition is provided that guarantees the inheritance of essential self-adjointness onto a certain class of restricted operators and allows us to conclude the self-adjointness of the reduced Laplace-Beltrami operators in a concise way. As a consequence, the self-adjointness of spin Calogero-Sutherland type reductions of 'free' Hamiltonians under polar actions of compact Lie groups follows immediately.
\end{abstract}

Keywords: Hamiltonian reduction, self-adjointness, polar action, integrable systems 


\section{Introduction}

The goal of this work is to verify the self-adjointness of certain reduced Hamiltonians obtained from quantum Hamiltonian reduction. The importance of the reduction method (see e.g. [1, 2]) mainly stems from the fact that under suitable symmetries the Hilbert space of a quantum system can be decomposed into invariant subspaces simplifying the diagonalization of the Hamiltonian. Another attractive perspective comes from the theory of integrable systems, since many integrable models arise as Hamiltonian reductions of canonical 'free' systems that are solvable due to their symmetries. The self-adjointness of the reduced Hamiltonians is in general necessary for the usefulness of the method.

If a compact Lie group $G$ acts smoothly on a complete Riemannian manifold $(Y, \eta)$ by isometries, then $G$ becomes a symmetry group of the quantum system of the free particle on $Y$. We take the free Hamiltonian to be just the Laplace-Beltrami operator, $\Delta_{Y}$, whose self-adjointness on a natural domain is guaranteed by the completeness of the underlying metric $\eta$. The system breaks up into $G$-invariant subspaces labeled by unitary irreducible representations $(\rho, V)$ of $G$, and the quantum Hamiltonian reduction gives rise to reduced Hamiltonians, $\Delta_{\rho}$, associated with these invariant subspaces.

In a recent paper [3], we studied quantum Hamiltonian reductions of Laplace-Beltrami operators on complete Riemannian manifolds under isometric actions of compact Lie groups that permit the introduction of generalized polar coordinates as defined in [4]. In this case we derived an explicit formula for the reduced Laplace-Beltrami operators and stated their essential selfadjointness on certain domains without detailed proof. Here, we provide the missing proof. Many examples of these reduced Hamiltonians yield integrable systems of spin Calogero-Sutherland type [5, 6, 7, 8, 9], and the present paper is part of our program aimed at exploring these interesting integrable systems in detail.

The content of the rest of the paper is as follows. In Section 2 we enquire whether the property of essential self-adjointness descends onto restrictions of essentially self-adjoint operators, and provide a sufficient condition under which essential self-adjointness of certain restrictions can be maintained. By using this condition, in Section 3 we prove the essential self-adjointness of the reduced Laplace-Beltrami operators in the general case of smooth isometric actions of compact Lie groups. We then apply this result to the important special case when $G$ acts on $Y$ in a polar manner in the sense of Palais and Terng [4]. In Section 4 we briefly recall from [3] the explicit formula of the reduced Laplace-Beltrami operators in the polar case, and confirm their essential self-adjointness. Finally, we give our conclusions in Section 5.

\section{Inheritance of essential self-adjointness upon restriction}

Let $A: \mathcal{D}(A) \rightarrow \mathcal{H}$ be a densely defined symmetric linear operator on a Hilbert space $(\mathcal{H},\langle\rangle$,$) .$ That is, the domain of $A, \mathcal{D}(A)$, is a dense linear subspace of $\mathcal{H}$, and $A$ satisfies the relation

$$
\left\langle A f_{1}, f_{2}\right\rangle=\left\langle f_{1}, A f_{2}\right\rangle \quad \forall f_{1}, f_{2} \in \mathcal{D}(A) .
$$


Let $S \subset \mathcal{H}$ be an invariant linear subspace of $A$, which means that $S \subset \mathcal{D}(A)$ and $A S \subset S$. These two assumptions guarantee that the restricted operator

$$
B:=\left.A\right|_{S}: S \rightarrow S
$$

is well defined. We can regard $B$ as a densely defined symmetric operator on the Hilbert space $\mathcal{M}:=\bar{S}$, where $\bar{S}$ denotes the closure of $S$ in $\mathcal{H}$.

Essentially self-adjoint operators admit unique self-adjoint extensions by their closures. It is easy to see that essential self-adjointness of $A$ does not imply, in general, the essential selfadjointness of the restricted operator $B=\left.A\right|_{S}$. Below we present a sufficient condition under which the property of essential self-adjointness descends onto the restricted operator.

Recall that the domain of the adjoint of $A$ is defined by the subspace

$$
\begin{array}{r}
\mathcal{D}\left(A^{*}\right)=\{g \mid g \in \mathcal{H} \text { for which } \exists k \in \mathcal{H} \text { such that } \\
\langle g, A f\rangle=\langle k, f\rangle \text { for every } f \in \mathcal{D}(A)\},
\end{array}
$$

and the adjoint of $A$ is the linear operator

$$
A^{*}: \mathcal{D}\left(A^{*}\right) \rightarrow \mathcal{H}, \quad g \mapsto A^{*} g:=k,
$$

where $k \in \mathcal{H}$ is the vector appearing in the definition of $\mathcal{D}\left(A^{*}\right)$. The 'deficiency subspaces', $L_{A}^{ \pm} \subset \mathcal{D}\left(A^{*}\right)$, of the operator $A$ are the sets

$$
L_{A}^{ \pm}:=\operatorname{Ker}\left(A^{*} \mp \mathrm{i}\right)=\left\{g \mid g \in \mathcal{D}\left(A^{*}\right), A^{*} g= \pm \mathrm{i} g\right\} .
$$

As is well known (see e.g. [10]), essential self-adjointness of a densely defined symmetric linear operator $A$ can be characterized by its deficiency subspaces $L_{A}^{ \pm}$. Namely, $A$ is essentially selfadjoint if and only if its deficiency subspaces are trivial, $L_{A}^{ \pm}=\{0\}$. We can consider the adjoint $B^{*}: \mathcal{D}\left(B^{*}\right) \rightarrow \mathcal{M}$ of the restricted operator $B(2)$, and its deficiency subspaces, too. Next we relate the adjoint of $B$ to the adjoint of $A$.

Lemma 2.1. Suppose that the domain of $A$ and the $A$-invariant linear subspace $S$ satisfy the following additional compatibility condition

$$
P_{\mathcal{M}} \mathcal{D}(A) \subset S
$$

where $P_{\mathcal{M}}: \mathcal{H} \rightarrow \mathcal{M}$ denotes the orthogonal projection onto the closed subspace $\mathcal{M}=\bar{S}$. Then $A^{*}$ is an extension of $B^{*}, B^{*} \subset A^{*}$, that is, $\mathcal{D}\left(B^{*}\right) \subset \mathcal{D}\left(A^{*}\right)$ and $\left.A^{*}\right|_{\mathcal{D}\left(B^{*}\right)}=B^{*}$.

Proof. First, take an arbitrary vector $f \in \mathcal{D}(A)$, and write it according to the orthogonal decomposition $\mathcal{H}=\mathcal{M} \oplus \mathcal{M}^{\perp}$ as $f=P_{\mathcal{M}} f+P_{\mathcal{M}^{\perp}} f$. Using the condition (6) we see that $P_{\mathcal{M}} f \in S \subset \mathcal{D}(A)$, therefore $P_{\mathcal{M}^{\perp}} f=\left(f-P_{\mathcal{M}} f\right) \in \mathcal{D}(A)$. Moreover, for every $u \in S$ we have $\left\langle u, A\left(P_{\mathcal{M}^{\perp}} f\right)\right\rangle=\left\langle A u, P_{\mathcal{M}^{\perp}} f\right\rangle=0$, whence we obtain $A\left(P_{\mathcal{M}^{\perp}} f\right) \in S^{\perp}=\mathcal{M}^{\perp}$.

Second, let $g \in \mathcal{D}\left(B^{*}\right)$ be an arbitrary vector. Then for every $f \in \mathcal{D}(A)$ we obtain

$$
\begin{gathered}
\langle g, A f\rangle=\left\langle g, A P_{\mathcal{M}} f+A P_{\mathcal{M}^{\perp}} f\right\rangle=\left\langle g, A\left(P_{\mathcal{M}} f\right)\right\rangle=\left\langle g, B\left(P_{\mathcal{M}} f\right)\right\rangle \\
=\left\langle B^{*} g, P_{\mathcal{M}} f\right\rangle=\left\langle B^{*} g, P_{\mathcal{M}} f\right\rangle+\left\langle B^{*} g, P_{\mathcal{M}^{\perp}} f\right\rangle=\left\langle B^{*} g, f\right\rangle .
\end{gathered}
$$


Therefore $g \in \mathcal{D}\left(A^{*}\right)$ and $A^{*} g=B^{*} g$.

Corollary 2.1. Under the above assumptions on $S$ and $\mathcal{D}(A)$, for the deficiency subspaces of the operators $A$ and $B=\left.A\right|_{S}$ we have the inclusion relations $L_{B}^{ \pm} \subset L_{A}^{ \pm}$, therefore if $A$ is essentially self-adjoint, so is its restriction $B$.

\section{Reduction of the Laplace-Beltrami operator}

Let $Y$ be a smooth, connected, complete Riemannian manifold with metric $\eta$. The restriction of the corresponding Laplace-Beltrami operator, $\Delta_{Y}$, onto the space of the complex valued compactly supported smooth functions,

$$
\Delta_{Y}^{0}:=\left.\Delta_{Y}\right|_{C_{c}^{\infty}(Y)}: C_{c}^{\infty}(Y) \rightarrow C_{c}^{\infty}(Y)
$$

is an essentially self-adjoint linear operator of the Hilbert space $L^{2}\left(Y, \mathrm{~d} \mu_{Y}\right)$, where $\mu_{Y}$ denotes the measure generated by the Riemannian volume form (see e.g. [1] and references therein). Suppose that a compact Lie group $G$ acts on $(Y, \eta)$ by isometries. More precisely, we are given a smooth left-action

$$
\phi: G \times Y \rightarrow Y, \quad(g, y) \mapsto \phi(g, y)=\phi_{g}(y)=g . y
$$

of $G$, such that $\phi_{g}^{*} \eta=\eta$ for every $g \in G$. The measure $\mu_{Y}$ inherits the $G$-invariance. Now take a finite dimensional continuous unitary irreducible representation $(\rho, V)$ of $G$, where $V$ is a complex vector space with inner product $(,)_{V}$. By simply acting componentwise, the operator $\Delta_{Y}^{0}$ extends onto the complex vector space of the $V$-valued compactly supported smooth functions, $C_{c}^{\infty}(Y, V)$. This gives the essentially self-adjoint operator

$$
\Delta_{Y}^{0}: C_{c}^{\infty}(Y, V) \rightarrow C_{c}^{\infty}(Y, V)
$$

of the Hilbert space $L^{2}\left(Y, V, \mathrm{~d} \mu_{Y}\right)$. Because of the $G$-symmetry of the metric $\eta$, the set

$$
C_{c}^{\infty}(Y, V)^{G}:=\left\{F \mid F \in C_{c}^{\infty}(Y, V), F \circ \phi_{g}=\rho(g) \circ F \quad \forall g \in G\right\}
$$

of the $V$-valued, compactly supported $G$-equivariant smooth functions is an invariant linear subspace of $\Delta_{Y}^{0}$, i.e., $C_{c}^{\infty}(Y, V)^{G} \subset C_{c}^{\infty}(Y, V)$ and $\Delta_{Y}^{0} C_{c}^{\infty}(Y, V)^{G} \subset C_{c}^{\infty}(Y, V)^{G}$.

Proposition 3.1. The restriction of $\Delta_{Y}^{0}$ (10) onto $C_{c}^{\infty}(Y, V)^{G}$,

$$
\Delta_{\rho}:=\left.\Delta_{Y}^{0}\right|_{C_{c}^{\infty}(Y, V)^{G}}: C_{c}^{\infty}(Y, V)^{G} \rightarrow C_{c}^{\infty}(Y, V)^{G},
$$

is a densely defined, symmetric, essentially self-adjoint linear operator on the Hilbert space $L^{2}\left(Y, V, \mathrm{~d} \mu_{Y}\right)^{G}$ of the square-integrable $G$-equivariant functions.

Proof. Notice that the closure of $C_{c}^{\infty}(Y, V)^{G}$ in $L^{2}\left(Y, V, \mathrm{~d} \mu_{Y}\right)$ equals $L^{2}\left(Y, V, \mathrm{~d} \mu_{Y}\right)^{G}$. Then it is enough to verify the compatibility condition (66), which in our case requires proving that $P_{\mathcal{M}} C_{c}^{\infty}(Y, V) \subset C_{c}^{\infty}(Y, V)^{G}$ with $\mathcal{M}:=L^{2}\left(Y, V, \mathrm{~d} \mu_{Y}\right)^{G}$. 
For each $F \in C_{c}^{\infty}(Y, V)$, let us define the function $\hat{F}: Y \rightarrow V$ by averaging over $G$,

$$
Y \ni y \mapsto \hat{F}(y):=\int_{G} \rho(g) F\left(g^{-1} \cdot y\right) \mathrm{d} \mu_{G}(g) \in V
$$

where $\mu_{G}$ denotes the (unique) bi-invariant probability Haar measure on $G$. It is easily seen that $\hat{F} \in C_{c}^{\infty}(Y, V)^{G}$. It also follows from (13) that the linear map

$$
P: C_{c}^{\infty}(Y, V) \rightarrow C_{c}^{\infty}(Y, V)^{G} \subset C_{c}^{\infty}(Y, V), \quad F \mapsto P F:=\hat{F}
$$

is a densely defined, symmetric, idempotent, and bounded operator on the Hilbert space $L^{2}\left(Y, V, \mathrm{~d} \mu_{Y}\right)$. The unique bounded extension of $P, \bar{P}: L^{2}\left(Y, V, \mathrm{~d} \mu_{Y}\right) \rightarrow L^{2}\left(Y, V, \mathrm{~d} \mu_{Y}\right)^{G}$, is just the orthogonal projection onto $\mathcal{M}=L^{2}\left(Y, V, \mathrm{~d} \mu_{Y}\right)^{G}$, i.e., $\bar{P}=P_{\mathcal{M}}$. As a consequence, we obtain the relations $P_{\mathcal{M}} C_{c}^{\infty}(Y, V)=P C_{c}^{\infty}(Y, V)=C_{c}^{\infty}(Y, V)^{G}$, proving the compatibility condition (6) for $A=\Delta_{Y}^{0}(10)$ and $S=C_{c}^{\infty}(Y, V)^{G}$.

Remark 3.1. The Hilbert space $L^{2}\left(Y, \mathrm{~d} \mu_{Y}\right)$ naturally carries a continuous unitary representation of $G$. This is unitarily equivalent to an orthogonal direct sum, $L^{2}\left(Y, \mathrm{~d} \mu_{Y}\right) \cong \oplus_{\rho} M_{\rho} \otimes V_{\bar{\rho}}$, where $\left(\rho, V_{\rho}\right)$ runs over a complete set of pairwise inequivalent irreducible continuous unitary representations of $G, \bar{\rho}$ denotes the complex conjugate of the representation $\rho$, and $M_{\rho}$ is a 'multiplicity space' on which $G$ acts trivially. Correspondingly, the self-adjoint scalar Laplace-Beltrami operator $\Delta_{Y}$, which by definition is the closure of $\Delta_{Y}^{0}$ in (8), can be decomposed as $\Delta_{Y} \cong \oplus_{\rho} \hat{\Delta}_{\rho} \otimes \mathrm{id}_{V_{\bar{\rho}}}$, where $\hat{\Delta}_{\rho}$ is a self-adjoint operator on the Hilbert space $M_{\rho}$. It is not difficult to demonstrate the unitary equivalence

$$
\left(M_{\rho}, \hat{\Delta}_{\rho}\right) \cong\left(L^{2}\left(Y, V, \mathrm{~d} \mu_{Y}\right)^{G}, \bar{\Delta}_{\rho}\right) \text { with } \quad V:=V_{\rho},
$$

where $\bar{\Delta}_{\rho}$ denotes the closure of $\Delta_{\rho}$ in (12). We find it convenient to use the realization of the reduced quantum system (15) furnished by $L^{2}\left(Y, V, \mathrm{~d} \mu_{Y}\right)^{G}$ (see also [2]).

\section{Explicit description of the reduced systems under polar actions}

We have seen that the reduced Hamiltonian entering the reduced quantum system (15) is provided by the essentially self-adjoint operator $\Delta_{\rho}(12)$. For purposes of interpretation, it would be desirable to realize the reduced state space $L^{2}\left(Y, V, \mathrm{~d} \mu_{Y}\right)^{G}$ as a Hilbert space of appropriate functions on the reduced configuration space $Y_{\text {red }}:=Y / G$, and the reduced Hamiltonian as a densely defined differential operator on this space. An apparent difficulty is that the orbit space $Y / G$ is not a smooth manifold but a stratified space in general, which among others means that $Y / G$ is a disjoint union of smooth manifolds of various dimensions. However, restricting to the principal orbit type $1, \check{Y} \subset Y$, one obtains a smooth fiber bundle $\pi: \check{Y} \rightarrow \check{Y} / G$ with fiber $G / K$ and structure

\footnotetext{
${ }^{1}$ We remind that $\check{Y}$ consists of those points $y \in Y$ whose isotropy subgroups, $G_{y}$, are the smallest possible for the given $G$-action; $\check{Y}$ is open and dense in $Y$. For reviews on group actions, stratifications and the principal orbit type, see e.g. [11, 12 .
} 
group $N_{G}(K) / K$, where $K \subset G$ is a closed subgroup representing the conjugacy class of principal isotropy subgroups, and $N_{G}(K)$ stands for the normalizer of $K$ in $G$. The 'big cell' of the reduced configuration space, given by $\check{Y}_{\text {red }}:=\check{Y} / G$, is naturally endowed with a Riemannian metric, $\eta_{\text {red }}$, making $\pi$ a Riemannian submersion. From a quantum mechanical point of view, neglecting the non-principal orbits is harmless, in some sense, since $\check{Y}$ is not only open and dense in $Y$, but it is also of full measure. Indeed, $\mu_{Y}(Y \backslash \check{Y})=0$ holds, since $Y \backslash \check{Y}$ is a union of at most countably many lower dimensional manifolds.

In many interesting applications of Hamiltonian reduction the group action is polar, which means that it admits sections in the sense of Palais and Terng [4]. Recall that a section $\Sigma \subset Y$ is a connected, closed, regularly embedded smooth submanifold of $Y$ that meets every $G$-orbit and it does so orthogonally at every intersection point of $\Sigma$ with an orbit. The induced metric on $\Sigma$ is denoted by $\eta_{\Sigma}$, and for the measure generated by $\eta_{\Sigma}$ we introduce the notation $\mu_{\Sigma}$. For a section $\Sigma$, denote by $\check{\Sigma}$ a connected component of the manifold $\hat{\Sigma}:=\check{Y} \cap \Sigma$. The isotropy subgroups of all elements of $\hat{\Sigma}$ are the same and for a fixed section we define $K:=G_{y}$ for $y \in \hat{\Sigma}$. By restricting $\pi: \check{Y} \rightarrow \check{Y} / G$ onto $\check{\Sigma},\left(\check{Y}_{\text {red }}, \eta_{\text {red }}\right)$ becomes identified with $\left(\check{\Sigma}, \eta_{\check{\Sigma}}\right)$, where $\eta_{\Sigma}$ is the induced metric on $\check{\Sigma}$. We let $\Delta_{\check{\Sigma}}$ stand for the Laplace-Beltrami operator of the Riemannian manifold $\left(\check{\Sigma}, \eta_{\check{\Sigma}}\right)$. The $G$-equivariant diffeomorphism

$$
\check{\Sigma} \times(G / K) \ni(q, g K) \mapsto \phi_{g}(q) \in \check{Y}
$$

provides a trivialization of the fiber bundle $\pi: \check{Y} \rightarrow \check{Y} / G$. The generalized polar coordinates on $\check{Y}$ consist of 'radial' coordinates on $\check{\Sigma}$ and 'angular' coordinates on $G / K$.

Below we characterize the reduced system (15) in terms of the reduced configuration space under the simplifying assumption of dealing with polar actions. We can be brief here as the details, except for the proof of the essential self-adjointness, can be found in our recent paper [3].

First, let us introduce the linear space

$$
\operatorname{Fun}\left(\check{\Sigma}, V^{K}\right):=\left\{f\left|f \in C_{c}^{\infty}\left(\check{\Sigma}, V^{K}\right), f=\mathcal{F}\right|_{\check{\Sigma}} \text { for some } \mathcal{F} \in C_{c}^{\infty}(Y, V)^{G}\right\},
$$

where $V^{K}$ is spanned by the $K$-invariant vectors in the representation space $V$. We assume that $\operatorname{dim}\left(V^{K}\right)>0$. The restriction of functions appearing in the definition (17) gives a linear isomorphism $\operatorname{Fun}\left(\check{\Sigma}, V^{K}\right) \cong C_{c}^{\infty}(Y, V)^{G} \hookrightarrow L^{2}\left(Y, V, \mathrm{~d} \mu_{Y}\right)^{G}$. This induces a scalar product on $\operatorname{Fun}\left(\check{\Sigma}, V^{K}\right)$ making it a pre-Hilbert space. The corresponding closure of Fun $\left(\check{\Sigma}, V^{K}\right)$ satisfies the Hilbert space isomorphism $\overline{\operatorname{Fun}}\left(\check{\Sigma}, V^{K}\right) \cong L^{2}\left(Y, V, \mathrm{~d} \mu_{Y}\right)^{G}$.

Next, consider the Lie algebra $\mathcal{G}:=\operatorname{Lie}(G)$ and its subalgebra $\mathcal{K}:=\operatorname{Lie}(K)$. Fix a $G$-invariant positive definite scalar product, $\mathcal{B}$, on $\mathcal{G}$, and thereby determine the orthogonal complement $\mathcal{K}^{\perp}$ of $\mathcal{K}$ in $\mathcal{G}$. For any $\xi \in \mathcal{G}$ denote by $\xi^{\sharp}$ the associated vector field on $Y$. Then at each point $q \in \check{\Sigma}$ the linear map $\mathcal{K}^{\perp} \ni \xi \mapsto \xi_{q}^{\sharp} \in T_{q} Y$ is injective, and the inertia operator $\mathcal{J}(q) \in \operatorname{End}\left(\mathcal{K}^{\perp}\right)$ can be defined by the requirement

$$
\eta_{q}\left(\xi_{q}^{\sharp}, \zeta_{q}^{\sharp}\right)=\mathcal{B}(\xi, \mathcal{J}(q) \zeta) \quad \forall \xi, \zeta \in \mathcal{K}^{\perp} .
$$

Note that $\mathcal{J}(q)$ is symmetric and positive definite with respect to $\left.\mathcal{B}\right|_{\mathcal{K}^{\perp} \times \mathcal{K}^{\perp}}$. By choosing dual bases $\left\{T_{\alpha}\right\},\left\{T^{\alpha}\right\} \subset \mathcal{K}^{\perp}$, that is, $\mathcal{B}\left(T^{\alpha}, T_{\beta}\right)=\delta_{\beta}^{\alpha}$, we let

$$
b_{\alpha, \beta}(q):=\mathcal{B}\left(T_{\alpha}, \mathcal{J}(q) T_{\beta}\right), \quad b^{\alpha, \beta}(q):=\mathcal{B}\left(T^{\alpha}, \mathcal{J}^{-1}(q) T^{\beta}\right) .
$$


We denote the representation of $\mathcal{G}$ corresponding to the representation $(\rho, V)$ of $G$ as $\rho^{\prime}: \mathcal{G} \rightarrow u(V)$, where $u(V)$ is the Lie algebra of anti-hermitian operators on $V$.

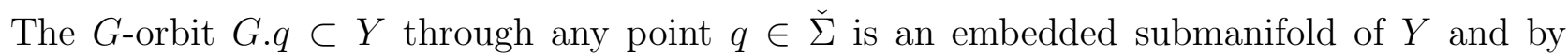
its embedding it inherits a Riemannian metric, $\eta_{G . q}$. Thus we can define the (smooth) density function $\delta: \check{\Sigma} \rightarrow(0, \infty)$ by

$$
\delta(q):=\text { volume of the Riemannian manifold }\left(G . q, \eta_{G . q}\right),
$$

where the volume is understood with respect to the measure, $\mu_{G \cdot q}$, belonging to $\eta_{G . q}$. If $\mu_{G / K}$ denotes the (unique) $G$-invariant probability Haar measure on $G / K$, then we have the relations $\mu_{G . q} \cong \delta(q) \mu_{G / K}$ and

$$
d \mu_{\check{Y}} \cong\left(\delta d \mu_{\check{\Sigma}}\right) \times d \mu_{G / K}
$$

between the various measures. Remember that $\check{Y} \cong \check{\Sigma} \times(G / K)$ by (16), and it is also worth pointing out that $\delta(q)=C\left|\operatorname{det} b_{\alpha \beta}(q)\right|^{\frac{1}{2}}$ with some constant $C>0$.

Proposition 4.1. Consider the reductions of the free particle on $(Y, \eta)$, with Hamiltonian given by $\Delta_{Y}^{0}$ (8), under a polar $G$-action. Then, using the above definitions, the reduced system (15) associated with a continuous unitary irreducible representation $(\rho, V)$ of $G$ can be identified with the pair $\left(L^{2}\left(\check{\Sigma}, V^{K}, \mathrm{~d} \mu_{\check{\Sigma}}\right), \Delta_{\text {red }}\right)$, where

$$
\Delta_{\text {red }}=\Delta_{\check{\Sigma}}-\delta^{-\frac{1}{2}} \Delta_{\check{\Sigma}}\left(\delta^{\frac{1}{2}}\right)+b^{\alpha, \beta} \rho^{\prime}\left(T_{\alpha}\right) \rho^{\prime}\left(T_{\beta}\right)
$$

with domain $\mathcal{D}\left(\Delta_{\text {red }}\right)=\delta^{\frac{1}{2}} \operatorname{Fun}\left(\check{\Sigma}, V^{K}\right)$ is a densely defined, symmetric, essentially self-adjoint operator on the Hilbert space $L^{2}\left(\check{\Sigma}, V^{K}, \mathrm{~d} \mu_{\check{\Sigma}}\right)$.

Proof. We have the Hilbert space identifications

$$
L^{2}\left(Y, V, \mathrm{~d} \mu_{Y}\right)^{G} \cong \overline{\operatorname{Fun}}\left(\check{\Sigma}, V^{K}\right) \cong L^{2}\left(\check{\Sigma}, V^{K}, \delta \mathrm{d} \mu_{\check{\Sigma}}\right),
$$

where the last equality follows from (21). We then consider the isometric isomorphism $U$ : $L^{2}\left(\check{\Sigma}, V^{K}, \delta \mathrm{d} \mu_{\check{\Sigma}}\right) \rightarrow L^{2}\left(\check{\Sigma}, V^{K}, \mathrm{~d} \mu_{\check{\Sigma}}\right)$ defined by the multiplication operator $U$ operating as $U: f \mapsto \delta^{\frac{1}{2}} f$. Using $\Delta_{\rho}(12)$ and the identifications (23) , in [3] we have established the explicit formula (22) for

$$
\Delta_{\text {red }} \equiv U \circ \Delta_{\rho} \circ U^{-1}
$$

Hence Proposition 3.1 implies that $\Delta_{\text {red }}$ is essentially self-adjoint on the domain obtained by transferring the domain of $\Delta_{\rho}$ into $L^{2}\left(\check{\Sigma}, V^{K}, \mathrm{~d} \mu_{\check{\Sigma}}\right)$ by means of (23) and the map $U$. The so-obtained domain is just $\delta^{\frac{1}{2}} \operatorname{Fun}\left(\check{\Sigma}, V^{K}\right)$.

Remark 4.1. The first term of formula (22) corresponds to the kinetic energy of a particle moving on $\left(\check{Y}_{\text {red }}, \eta_{\text {red }}\right) \cong\left(\check{\Sigma}, \eta_{\check{\Sigma}}\right)$ and the rest represents potential energy if $\operatorname{dim}\left(V^{K}\right)=1$ (which happens very rarely [6, 7]). The second term of (22) is of course always potential energy (and in certain cases just a constant). If $\operatorname{dim}\left(V^{K}\right)>1$, then one says that the reduced system contains internal 'spin' degrees of freedom and the last term of (22) encodes spin-dependent potential energy. For comparison with the result of classical Hamiltonian reduction, see [3] and references therein. 


\section{Conclusion}

In this work we focused our attention on the inheritance of the (essential) self-adjointness property in the process of quantum Hamiltonian reduction. By using the auxiliary result of Section 2, we proved in Section 3 the essential self-adjointness of the reduced Laplace-Beltrami operators (12) on complete Riemannian manifolds equipped with smooth isometric actions of compact Lie groups. Lemma 2.1 can be applied in other examples as well to prove essential self-adjointness for reduced Hamiltonians.

Assuming that the symmetry group acts in a polar manner, in Section 4 we recalled the explicit realization (22) of the reduced Laplace-Beltrami operators in terms of the reduced configuration space, and complemented the results of [3] by verifying the essential self-adjointness of these operators. It is worth noting that if the underlying Riemannian manifold is itself a Lie group, or a symmetric space, and the 'sections' can be realized as flat Abelian subgroups, then the Hamiltonian reductions of the Laplace-Beltrami operator typically yield spin Calogero-Sutherland type models (see e.g. [5, 6, 7, 8, 9]). Many known and new (non-elliptic) spin Calogero-Sutherland type models arise in this framework and certain spinless cases also appear among the reduced systems. We shall further elaborate the structure of these models in the future.

Acknowledgments. The work of L.F. was supported by the Hungarian Scientific Research Fund (OTKA grant T049495) and by the EU network 'ENIGMA' (contract number MRTN-CT-20045652). B.G.P. is grateful to J. Harnad for hospitality in Montréal.

\section{References}

[1] N.P. Landsman: Mathematical Topics Between Classical and Quantum Mechanics, Springer, New York 1998

[2] S. Tanimura and T. Iwai: Reduction of Quantum Systems on Riemannian Manifolds with Symmetry and Application to Molecular Mechanics, J. Math. Phys. 41, 1814-1842 (2000), arXiv:math-ph/9907005

[3] L. Fehér and B.G. Pusztai: Hamiltonian reductions of free particles under polar actions of compact Lie groups, arXiv:0705.1998 [math-ph] (to appear in Theor. Math. Phys.)

[4] R. Palais and C.-L. Terng: A general theory of canonical forms, Trans. Amer. Math. Soc. 300, 771-789 (1987)

[5] M.A. Olshanetsky and A.M. Perelomov: Quantum integrable systems related to Lie algebras, Phys. Rept. 94, 313-404 (1983)

[6] P.I. Etingof, I.B. Frenkel and A.A. Kirillov Jr.: Spherical functions on affine Lie groups, Duke Math. J. 80, 59-90 (1995), arXiv:hep-th/9407047

[7] A. Oblomkov: Heckman-Opdam's Jacobi polynomials for the $B C_{n}$ root system and generalized spherical functions, Adv. Math. 186, 153-180 (2004), arXiv:math.RT/0202076 
[8] L. Fehér and B.G. Pusztai: Spin Calogero models obtained from dynamical r-matrices and geodesic motion, Nucl. Phys. B734, 304-325 (2006), arXiv:math-ph/0507062

[9] L. Fehér and B.G. Pusztai: A class of Calogero type reductions of free motion on a simple Lie group, Lett. Math. Phys. 79, 263-277 (2007), arXiv:math-ph/0609085

[10] M. Reed and B. Simon: Methods of Modern Mathematical Physics, Vol. II, Academic Press, New York 1975

[11] M. Davis: Smooth G-manifolds as collections of fiber bundles, Pacific J. Math. 77, 315-363 (1978)

[12] V.V. Gorbatsevich, A.L. Onishchik and E.B. Vinberg: Foundations of Lie Theory and Lie Transformation Groups, Springer, Berlin 1997 